\title{
CT and MRI findings of anthrax meningoencephalitis; Case report
}

\author{
Sercan Özkaçmaz \\ Clinic of Radiology, Van Education and Training Hospital, Van, Turkey
}

\begin{abstract}
Anthrax meningoencephalitis is a rare but mortal condition of anthrax. Primary focus of infection may be skin, respiratory and gastrointestinal anthrax. It has a poor prognosis with fatal outcome despite aggressive combined antibiotic therapy. Radiological findings demonstrate a hemorrhagic encephalitis pattern with cerebral edema. We present an anthrax meningoencephalitis case with CT, MRI and DWI findings from an endemic region.
\end{abstract}

Key Words: Anthrax, meningoencephalitis, imaging findings

\section{Introduction}

Anthrax is a zoonotic infection which spreads by handling or eating infected product of infected animals and by inhalation of Bacillius anthrax spores (1). Meningoencephalitis is a rare clinical condition of anhtrax which almost has an aggressive clinical course and fatal outcome despite aggressive antibiotic therapy and intensive care supports. Anthrax is diagnosed by detecting B. anthracis in samples taken from blood, spinal fluid, skin lesions, or respiratory secretions and by growth of bacteria in cultures $(1,2)$.

Although this progressive radiological and clinical antity mostly occurs secondary to skin, pulmonary and gastrointestinal involvement through hematogenous and lymphogenous route, in rare cases primary source cannot be detected.

Anthrax meningoencephalitis occurs in less than $5 \%$ of all patients with anthrax (3). Most common symptom and findings are nonspecific which include headache, confusion, muscle weakness, seizures, neck rigidity, delirium and coma. Although a history of handling or eating meats of infected animals, high-risked jobs such as butchers, progressive neurological symptoms and living in epidemic areas may be suggestive for anthrax menengoencephalitis (3).

Anthrax meningoencephalitis usually exhibits as hemorrhagic encephalitis. Treatment of the disease is composed of combined antibiotic therapy and intensive care.

As it is a fatal disease and common in eastern of our country we present a primary source unknown anthrax meningoencephalitis case with CT and MRI findings.

\section{Case report}

A 22 years old male admitted to emergency department with fatigue and a severe headache for 6 hours. His medical history was remarkable. A physical examination revealed confusion with mild both upper and lower extremity weaknesses. CT scan at the admission was normal except for a mild cerebral edema. He had no respiratory distress by this time. At admission, his temperature was $35.5^{\circ} \mathrm{C}$, respiration rate was 24 , hemoglobin was $12.69 \mathrm{~g} / \mathrm{dL}$, white blood cell count was $23500 / \mathrm{mm}^{3}$, erythrocyte sedimentation rate was $8 \mathrm{~mm} / \mathrm{h}$, and fibrinogen was 3.1 , AST was 140 and ALT was 117.

When he was evaluating for a possible encephalitis and venous sinus thrombosis diagnosis in neurology clinic, 12 hours after admission, suddenly he became comatose, and required intubation, then transferred to intensive care department. On DWI at this time, there was no restriction in brain. Blood culture was positive for diphtheroid bacilli. A Lumbar puncture showed a white blood count of $2250 / \mathrm{mm}^{3} \quad(80 \%$ neutrophils), and the CFS was bloody. The protein was 9461, the glucose was 107, chloride was 118. Growth of Bacillus anthracis was observed in cerebrospinal fluid (CSF) culture (Figure 1). On his detailed skin and oropharyngeal 


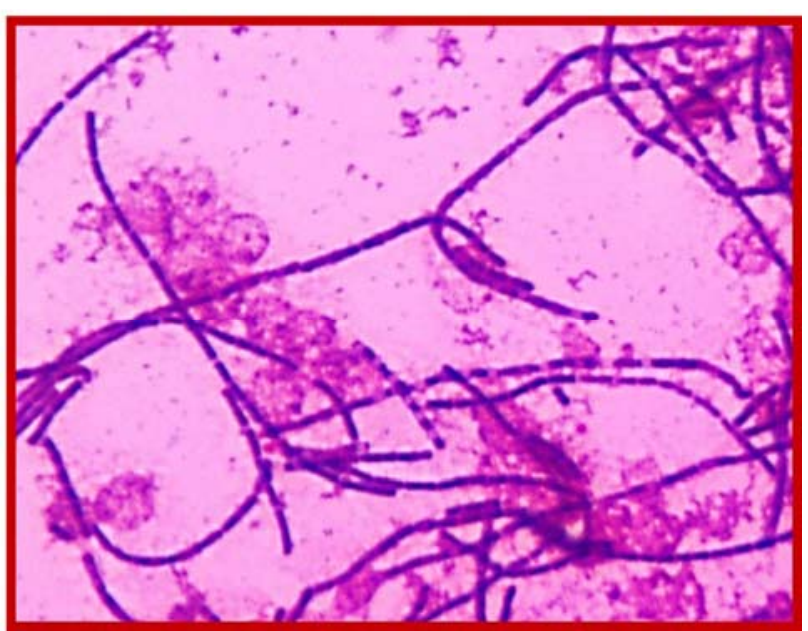

Fig. 1. Growth of Bacillus anthracis was observed in CSF culture. examination there was no any lesion compatible with anthrax involvement. No any bacilli was demonstrated on stool and sputum samples. He had no respiratory stress and also mediastinal widening or pleural effusion which may be suggestive for pulmonary anthrax, were not detected on chest roentgenograms.

On the second day of hospitalisation, a nonenhanced conventional brain MRI and a venous MR Angiography demonstrated involvements of bilaterally caudate nuclei and frontal and parietal lobes which were compatible with encephalitis without sinus venous thrombosis (Figure 2a,b). Also DWI showed cortical restriction in bilaterally frontal and parietal lobes (Figure 3).

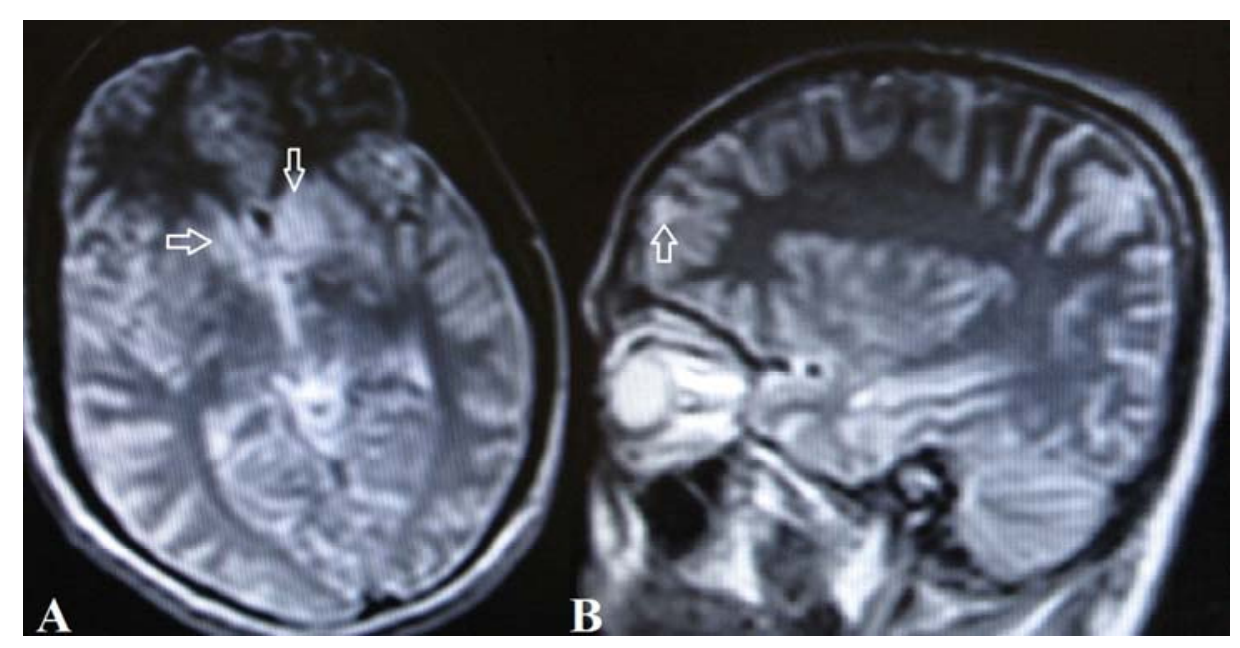

Fig. 2a,b. (a) Axial T2 weighted image shows bilaterally hyperintensities on caudat nuclei, and (b) Sagittal T2 weighted image demonstrates cortical hyperintensities on left frontoparietal region (arrows).

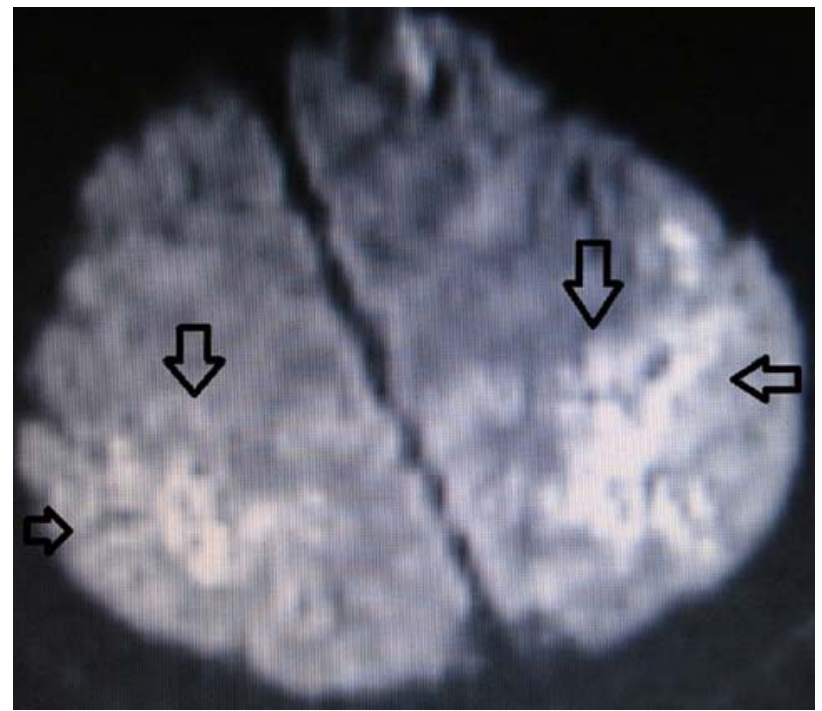

Fig. 3. DWI demonstrates cortical restriction in bilateral frontoparietal lobes.
A diagnosis of anthrax menengoencephalitis was made and a combination of Penicillin G, Vancomycin, Rifampicin and anti edema treatment were started. On the 4th Day of hospitalisation his general medical status worsened. A brain CT by this time revealed hydrocephalus, severe brain edema and linear hemorrhages along gyral surfaces (Figure 4a,b). Despite aggressive treatment patient died on the 5 th Day.

\section{Discussion}

Cutaneous anthrax is endemic in Turkey, especially in Eastern region $(1,2)$. Meningoencephalitis is a rare condition of anthrax. Although the frequency of this condition is not well demonstrated, some studies report meningoencephalitis as less than $5 \%$ of all anthrax

East J Med Volume:22, Number:3, July-September/2017 


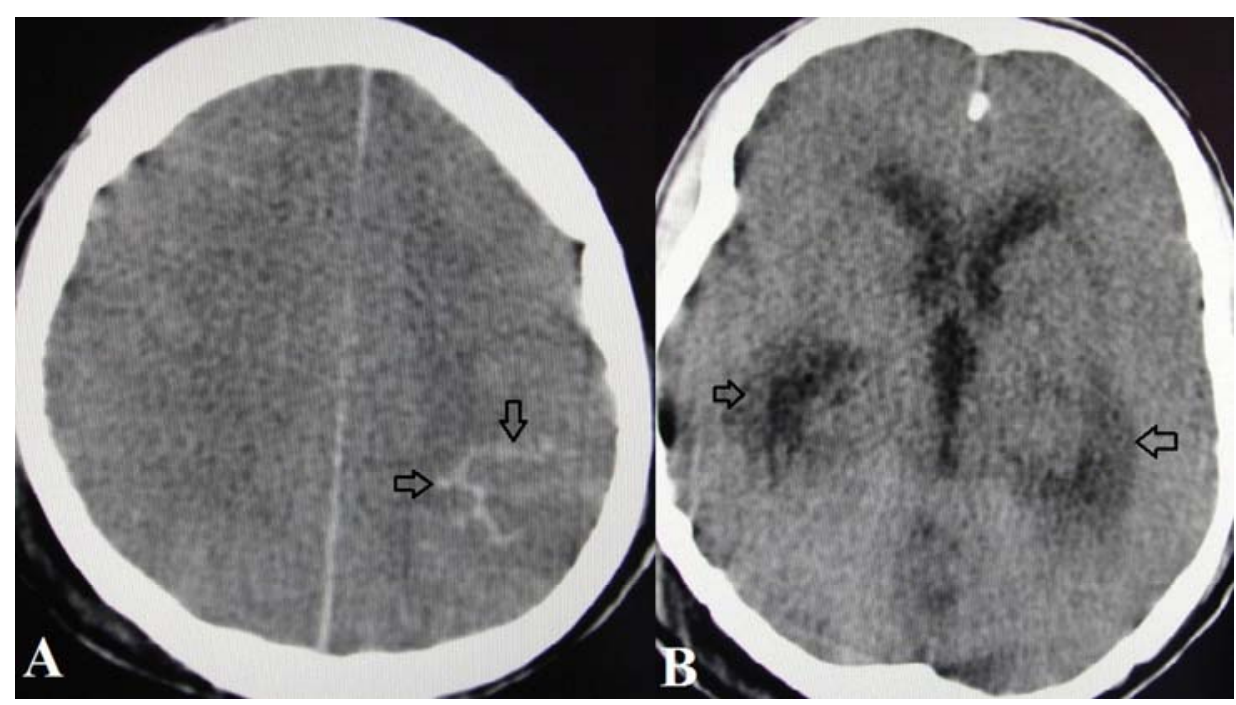

Fig. 4a, b. (a) Non-enhanced brain CT shows subarachoid hemaorhagies on left frontoparietal region, and (b) Non-enhanced brain CT demonstrates hydrocephalus with severe edema.

cases $(1,3)$. To now, about 100 cases with anthrax menengoencephalitis were reported (4-17). Lanska et al. (18) reviewed 70 cases with anthrax menengoencephalitis between 1952 and 2002. In our country 8 cases with anthrax mengoencephalitis was reported between 19902009 (7,11-17). Lanska et al. (18) investigated the sources of anthrax menengoencephalitis in their 70 patients, too. Sources of menengoencephalitis in anthrax were respiratory in 39\%, cutaneous in $29 \%$, and gastrointestinal in $17 \%$ of these patients. No source was identified in $16 \%$ of them. The primary sources were cutaneous in 5 cases, respiratory in one case of the 8 patients with anthrax meningoencephalitis reported in Turkey. With two of them the source was not identified (7,11-17). Relatives of our patient reported that he ate meat from a sick cow before a few days in their village. Based on this history the source was suggested as gastrointestinal anthrax.

CSF in anthrax meningoencephalitis may appear cloudy, with yellowish or pinkish coloration or may be grossly bloody. CSF showes increased white blood cell count with predominantly polymorphonuclear leukocytes, decreased glucose level, and elevated protein levels in anthrax menengoencephalitis (18). In our patient CSF protein was $9461 \mathrm{mg} / \mathrm{dL}$, chloride was 118 $\mathrm{mmol} / \mathrm{L}$ and glucose was $107 \mathrm{mg} / \mathrm{dL}$ (Simultaneous blood glucose level was 226 $\mathrm{mg} / \mathrm{dL}$ ), white blood cell count was $2250 / \mathrm{mm}^{3}$ (80\% neutrophils) and CFS was bloody.

The diagnosis of anthrax meningoencephalitis is made when gram positive encapsulated bacilli are seen in CSF and growth of this bacteria in cultures. Polymerase chain reaction (PCR) is an important method for a rapid and accurate diagnosis of anthrax. Gram staining of the CSF showed gram positive bacilli and CFS culture revealed anthrax growth in our patient. Also CSF from lumbar puncture was positive for B. anthracis DNA by PCR Growth of the anthrax bacilli in CSF cultures is accepted as gold standard but it can take time (3).

The major neurological complication of anthrax meningoencephalitis is mortal subarachnoid hemorrhages (5-7,9-11). In the literature, the most common histopathological findings of anthrax meningoencephalitis are necrotizing vasculitis, cerebral infarction, edema, hemorrhagic meningitis, intraventricular hemorrhage, multifocal subarachnoid or intraparenchymal hemorrhages (3). Hemorrhagic meningitis was detected in 6 of 8 patients with anthrax meningoencephalitis which were reported in Turkey (7,14-17). Also hemorrhagic meningitis and subarachnoid hemorrhage were observed in our patient.

Chest x-ray findings included pleural effusion, hilar adenopathy, widened mediastinum, infiltrates, and soft tissue edema (18).

CT or MRI of the head in patients with anthrax meningoencephalitis may demonstrate focal intracerebral hemorrhage, subarachnoid hemorrhage, intraventricular hemorrhage, diffuse cerebral edema, and prominent leptomeningeal enhancement. Parenchymal cerebral enhancement 
has not been reported (18). The subarachnoid space, deep gray matter, and ventricle were also reported as the sites of hemorrhage in patients with anthrax meningoencephalitis. In addition, prominent diffuse leptomeningeal enhancement was shown in this study (19). Similar findings were reported in the patients with anthrax meningoencephalitis in literature $(7,11,13)$. Compatible with previous reports, we detected brain edema, subarachnoid hemorrhages and hydrocephalus in our patient.

Because of progressive course and fatal outcome, early diagnosis and appropriate antibiotic therapy is critical for reducing the mortality rates. Despite aggressive treatment, success rates are low. Also delayed diagnosis and treatment decrease these rates.

In conclusion, prognosis is poor and mortality rate is high for the central nervous system infections due to $\mathrm{B}$. anthrax. It must be kept in mind for hemorrhagic encephalitis in endemic regions such as Turkey even the patient has not a skin lesion and has not history of exposure to products of infected animals.

\section{References}

1. Doğanay $M$, Metan G. Human anthrax in Turkey from 1990 to 2007. Vector Borne Zoonotic Dis 2009; 9: 131-140.

2. Karahocagil MK, Akdeniz N, Akdeniz $\mathrm{H}$ et al. Cutaneous anthrax in Eastern Turkey: a review of 85 cases, Clin Exp Dermatol 2008; 33: 406411.

3. Doğanay M. Bacillus anthracis and other Bacillus species. In: Topcu AW, Soyletir G, Doğanay M, editors. Infectious diseases and their microbiology. Istanbul: Nobel Press; 2008. p.2102-14.

4. Babamahmoodi F, Aghabarari F, Arjmand A, Ashra H. Three rare cases of anthrax arising from the same source. J Infect 2006; 53: e175e179.

5. Khoddami M, Shirvani F, Esmaeili J, Beladimogaddam N. Two rare presentations of fatal anthrax: meningeal and intestinal. Arch Iran Med 2010; 13: 432-435.
6. Kwong KL, Que TL, Wong SN, So KT. Fatal meningoencephalitis due to Bacillus anthracis. J Paediatr Child Health 1997; 33: 539-541.

7. Gurcan S, Akata F, Kuloglu F, Erdogan S, Tugrul M. Meningitis due to bacillus anthracis. Yonsei Med J 2005; 46: 159-160.

8. Khanna N, Gokul BN, Ravikumar R, et al. Successfully treated primary anthrax meningitis. Indian J Pathol Microbiol 1989; 32: 315-317.

9. Meyer MA. Neurologic complications of anthrax. A review of the literature. Arch Neurol 2003; 60: 483-488.

10. Bharathmoorthy, Chakravarthy I, Swaminathan $\mathrm{RP}$, et al. Hemorrhagic meningitis due to Bacillus anthrax. J Assoc Physicians India 1992; 40: 134-135.

11. Leblebicioglu H, Turan D, Eroglu C, et al. A cluster of anthrax cases including meningitis. Trop Doc 2006; 36: 51-53.

12. Kilıçturgay K, Gökırmak F, Töre O, Göral G, Helvacı S. Bir şarbon menenjiti vakası. Uludağ Üniversitesi Tıp Fakültesi Derg 1985; 1-3: 7375.

13. Yorgancigil B, Demirci M, Unlu M, Sevük E, Doganay M. Anthrax meningitis: case report. Int J Infect Dis 2001; 5: 220-221.

14. Albayrak F, Memikoglu O, Kurt O, et al. A case of anthrax meningitis. Scand J Infect Dis 2002; 34: 627-628.

15. Tasyaran MA, Deniz O, Ertek M, Cetin K. Anthrax meningitis: case report and review. Scand J Infect Dis 2002; 34: 66-67.

16. Yakupogullar1 Y, Kabakus N, Durukan M, et al. Anthrax meningoencephalitis secondary to oral infection. Pediatr Infect Dis J 2006; 25: 572-573.

17. Metan G, Uysal B, Coskun R, Percin D, Doğanay M. Anthrax meningoencephalitis: a case report and review of Turkish literature. Mikrobiyol Bul 2009; 43: 671-676.

18. Lanska DJ. Anthrax meningoencephlitis. Neurology 2002; 59: 327-334.

19. Kim HJ, Jun WB, Lee SH, Rho MH. CT and MR findings of anthrax meningoencephalitis: report of two cases and review of the literature. AJNR Am J Neuroradiol 2001; 22: 1303-1305. 